\title{
OPTIMAL RISK EXCHANGES *
}

\author{
Hans Bühlmann and William S. Jewell
}

\begin{abstract}
The determination of optimal rules for sharing risks and constructing reinsurance treaties has important practical and theoretical interest. Medolaghi, de Finetti, and Ottaviani developed the first linear reciprocal reinsurance treaties based upon minimizing individual and aggregate variance of risk. Borch then used the economic concept of utility to justify choosing Pareto-optimal forms of risk exchange; in many cases, this leads to familiar linear quota-sharing of total pooled losses, or to stop-loss arrangements. However, this approach does not give a unique, risk-sharing agreement, and may lead to substantial fixed side payments. Gerber showed how to constrain a Pareto-optimal risk exchange to avoid invasion of reserves.

To these ideas, the authors have added the actuarial concept of long-run fairness to each participant in the risk exchange; the result is a unique, Pareto-optimal risk pool, with "quota-sharing-by-layers" of the total losses. There are many interesting special cases, especially when all individual utility functions are of exponential form, giving linear quota-sharing-by-layers. Algorithms and numerical examples are given.
\end{abstract}

\section{INTRODUCTION}

Insurance companies and other financial risk-bearing entities may enter into formal risk-sharing agreements for a variety of reasons, the most important of which is the simultaneous reduction of risk for all participants. For example, it is well known that two companies can both reduce the variance of their risk portfolios by agreeing to cover fixed quotas $(\beta, 1-\beta)$ of their pooled losses (and perhaps making a side payment to keep the pool "on fair terms"); this joint improvement occurs for some interval of values of $\beta$ in $(0,1)$, so the actual quota must be negotiated by other considerations.

Other corporate objectives, such as market penetration or financial stability may lead to different, non-linear forms of exchange, in which extreme, catastrophic losses are reallocated to the treaty members in different ways to "spread the risk". In fact, there is no difficulty in including under risk exchanges such "one-sided" arrangements as a reinsurance treaty, in which one of the participants brings no risks to the pool, but agrees to take a portion of the excess losses above some retention limit, in exchange for a fixed fee. Clearly it would be desirable to develop a theory which would explain the variety of actual risk-sharing agreements observed in the real world.

Given that a group of insurance companies has agreed to enter into a risk pool, this paper explores the general forms of exchange that result in simultaneous improvement of risk for all parties, under the following assumptions:

* Presented at the I4th ASTIN Colloquium, Taormina, October I978. 
1. All companies share the same information about the statistical nature of the individual, possibly dependent, risks to be shared. We formalize this by assuming that all companies work with the same probability distributions of the risks concerned.

2. Each company measures the risk of its portfolio by an individual utility function that is unaffected by the negotiations, i.e. there is no effort to change attitudes.

3. The companies may also agree, through mechanisms not considered here, on certain individual or joint side conditions, such as limits on losses paid, or on side payments.

In a series of important papers (1960a, 1960b, 1962), Borch showed that the use of utility functions leads to the economic concept of Pareto-optimal risk exchanges, in which the form of the agreements is determined by the individual utility functions (however not by the probability distribution!). Solution parameters are still open to competitive negotiations. In this paper we shall add the assumption that

4. all companies wish the exchange to be fair, in the sense that, according to a commonly accepted premium principle, all companies agree that, over the long run, no company in the pool should profit at the expense of the others.

We shall see that adding this insurance concept of fairness will lead to a unique Pareto optimal risk-sharing agreement. Many familiar forms of exchanges then follow under special assumptions about utility functions, volume of business, individual participation constraints, etc.

It is interesting that so far all authors have tried to arrive at a specific element within the set of Pareto Optima by game theoretic considerations (Borch (1960b), Lemaire (1977)). Our paper achieves unicity by introducing the actuarial concept of fairness.

\section{THE MODEL}

\subsection{General Considerations}

Consider $n$ insurance companies, indexed $i=1,2, \ldots, n$, each with a risk portfolio characterized by

- a fixed premium income, $\Pi_{i} \geqslant 0$;

- a random loss (possible claims), $S_{i} \geqslant 0$;

over some common exposure period. The set of all losses $\left[S_{i}\right]$ is defined over some probability space $\Omega$ with known joint distribution $P(\omega)$ of possible outcomes $\omega$. Set $\Pi=\Sigma \Pi_{i}$ and $S=\Sigma S_{i}$. 
By a risk exchange or risk pool we mean any formal mutual agreement among the $n$ companies that, operating as an entity

1. accepts the responsibility for paying for an input $X_{i}=f_{i}\left(S_{i}, I_{i}\right)$ from company $i$, where $f_{i}$ is a fixed but arbitrary function;

2. charges company $i$ an output $Y_{i}$ for accepting the input, according to the agreed-upon rule for sharing risks;

3. Operates on a zero-balance conservation principle

$$
\Sigma Y_{i}(\omega)=\Sigma X_{i}(\omega)=X(\omega)
$$

for all outcomes $\omega \in \Omega$.

Since risk pools are intended to redistribute only actual losses (and possibly the associated premiums), but not the individual wealth of the company, one would typically include in the exchange protocol side constraints of the form

$$
Y_{i} \geqslant A_{i} \quad(i=1,2, \ldots, n)
$$

where $A_{i}$ is a constant (or random variable $A_{i}(\omega)$ ), designed to limit negative charges (payouts) to company $i$.

The risk agreements might also include constraints of the type

$$
Y_{i} \leqslant A_{i}+B_{i}, \quad(i=1,2, \ldots, n),
$$

designed to directly protect the liquidity of the individual companies. It is clear that we must have $B_{i} \geqslant 0$. We shall not consider pools in which coalition constraints, relating to subsets of the $Y_{i}$, are possible. The importance of side constraints is mentioned by Borch (1968), and first incorporated in an exchange model by Gerber (1978).

\subsection{The Claims Pool; Linear and Quota Exchanges}

An important special case is the claims pool in which companies keep their premiums and share all their losses:

$$
X_{i}=S_{i} ; Y_{i} \geqslant 0, \quad(i=1,2, \ldots, n)
$$

One possible risk-sharing rule is a linear exchange, in which $n^{2}$ constants $\theta_{i j}$ are given, so that

$$
Y_{i}=\sum_{j=1}^{n} \theta_{i j} X_{j}, \quad(i=1,2, \ldots, n)
$$

To satisfy the clearing condition $\Sigma Y_{i}=\Sigma X_{i}$, we must have

$$
\sum_{i=1}^{n} \theta_{i j}=1, \quad(j=1,2, \ldots, n) \text {, }
$$

so that a feasible linear exchange has only $n^{2}-n$ free constants, $0 \leqslant \theta_{i j} \leqslant 1$. 
If the losses $X_{i}$ are uncorrelated, it is easy to show (for $n=2$, see BEARD (1969)) that the variance of all output losses $V\left\{Y_{i}\right\}$ can be reduced by adjusting the constrained $\theta_{i j}$ until a Pareto-optimal frontier is reached at which a variance reduction for one company must be traded-off against a variance increase for another. On this frontier, there are only $n$ exchange constants, $\theta_{i j}=\theta_{i}$, and

$$
Y_{i}=\theta_{i} X ; 0 \leqslant \theta_{i} \leqslant 1 ; \Sigma \theta_{i}=1 .
$$

This arrangement is called a quota claims pool; the quota fraction $\theta_{i}$, taken by the $i$ th company of the total losses $S$, is often fixed on the basis of "volume", e.g.,

$$
\theta_{i}=\Pi_{i} / \Pi \text {. }
$$

Note that one could add conservative side payments to (2.5) to make $E\left\{Y_{i}\right\}=E\left\{X_{i}\right\}$ without changing the variances of the quota claims pool. The first models of linear quota pools were developed by P. Medolaghi, B. de Finetti, and G. Ottaviani; references are given in SEAL (1969).

\subsection{The Business Pool}

Another important special case is when companies agree to share both premiums and claims:

$$
X_{i}=S_{i}-\Pi_{i} ; Y_{i} \geqslant-\Pi, \quad(i=1,2, \ldots, n) .
$$

Notice the lower limit to prevent the invasion of reserves.

The traditional quota business pool is a linear exchange of this type, in which

$$
Y_{i}=\theta_{i}(S-\Pi) ; 0 \leqslant \theta_{i} \leqslant 1 ; \Sigma \theta_{i}=1 .
$$

Observe that if these quotas are set on the basis of "volume" (2.8), then the quota claims and quota business pools coincide, in the sense that the net charge to company $i, Y_{i}-X_{i}$, is the same in both cases.

\subsection{The Canonical Risk Exchange (REX)}

It is easy to see that if both $X_{i}$ and $Y_{i}$ are changed by the same (possibly random) amount, the net charges of a risk pool remain the same. To simplify exposition in the sequel, we shall subtract out any $A_{i}$ appearing in (2.2) from the definitions of $X_{i}$ and $Y_{i}$ in (2.1), giving $X_{i}=f_{i}\left(S_{i}, \Pi_{i}\right)-A_{i}$ and $Y_{i} \geqslant 0$. This will also affect any other side conditions, such as (2.3).

Our canonical definition will then be:

A risk exchange (REX) $(X, Y)$ is a formal rule for changing a random vector $X=\left(X_{1}, X_{2}, \ldots, X_{n}\right)$ into a random vector $Y=\left(Y_{1}, Y_{2}, \ldots, Y_{n}\right)$ so that

$$
\text { 1. } \Sigma Y_{i}=\Sigma X_{i} \text {. }
$$


(2.11b)

2. $Y_{i} \geqslant 0, \quad(i=1,2, \ldots, n)$.

$(2.11 \mathrm{c})$

3. $Y_{i} \leqslant B_{i}, \quad(i=1,2, \ldots, n)$.

\section{THE FAIR RISK EXCHANGE (FAIRREX)}

Most insurance business is carried out using a measure of risk called premium. If $Z$ is any random variable defined on $\Omega$, we may define a premium principle for $Z$ as follows:

$$
\text { Premium }[Z]=\int_{\Omega} Z(\omega) G(\omega) d P(\omega)
$$

$G(\omega)>0$ is a loading factor that weighs $Z$ in a predetermined manner over the possible outcomes. If $G$ is the random variable with values $G(\omega)$, we can also write Premium $[Z]=E\{Z G\}$. Typically, $E\{G\} \geqslant 1$; for later purposes, we require $G>0$ on the support of $Z$. Finally, if $G=1$ for all $\omega$, then (3.1) gives the usual fair premium $E\{Z\}$.

In establishing a risk pool, particularly a mutual agreement among similar companies, we argue that no company should profit from any other in the long run, no matter what form of agreement is mutually best for the outcomes in each exposure period. This implies that each company, using a commonly accepted premium principle, would judge that the premium of its input to the pool should be identical with the premium of the output it actually pays, in order that the pool is perceived to be equitable in the long run.

Therefore we define:

A $\operatorname{REX}(X, Y)$ is a FAIRREX if it satisfies the fairness condition

$$
\operatorname{Premium}\left[Y_{i}\right]=\operatorname{Premium}\left[X_{i}\right]
$$

for each company $(i=1,2, \ldots, n)$, using a common premium principle (3.1). Note that for all values of $\theta_{i}$ the quota business pool (2.9) is a FAIRREX if $\Pi_{i}$ is the premium calculated on a fair premium basis. Unfortunately the linear form of exchange is not usually optimal in the sense described in the next section.

\section{THE PARETO-OPTIMAL RISK EXCHANGE (POREX)}

\subsection{The Unconstrained Case}

Borch (1960a, 1960b, 1962) observed that the form of treaty acceptable to all parties in a REX should depend upon the individual attitudes towards risk. Assuming that each company is rational in ordering its preferences (i.e., satisfies the Bernoulli hypothesis); then it is well-known that under weak technical assumptions this implies the existence of a non-decreasing utility function $u_{i}$ for each company $(i=1,2, \ldots, n)$, and the ranking of risky outcomes according to its expected utility $U_{i}$. For example, suppose that, prior to 
joining a REX, company $i$ held capital wealth $W_{i}$ against a random loss $X_{i}$. Its prior expected utility would be

$$
U_{i}^{0}=E\left\{u_{i}\left(W_{i}-X_{i}\right)\right\}
$$

Posterior to the REX (2.11), its expected utility will be

$$
U_{i}=E\left\{u_{i}\left[W_{i}-Y_{i}\left(X_{1}, X_{2}, \ldots, X_{n}\right)\right]\right\},
$$

and so it will perceive the REX as advantageous for company $i$, if $U_{i}>U_{i}^{0}$.

Borch argued that if the companies acted rationally and cooperatively, they would not agree on a $\operatorname{REX}(X, Y)$ if there existed another $\operatorname{REX}(X, \widetilde{Y})$ such that the expected utility of at least one company was improved without decreasing the expected utility of all other participants. This leads naturally to the idea that the all interesting treaties are Pareto-optimal, defined as

A $\operatorname{REX}(X, Y)$ is a Pareto-Optimal Risk Exchange if there is no other $\operatorname{REX}(X, \ddot{Y})$ with

$$
E\left\{u_{i}\left(W_{i}-\widetilde{Y}_{i}\right)\right\} \geqslant E\left\{u_{i}\left(W_{i}-Y_{i}\right)\right\}
$$

for all $i$, with strict inequality for at least one $i$.

Since we will be dealing only with non-negative losses, we can simplify our formulae by changing to disutility functions $v_{i}$ and expected disutilities $V_{\boldsymbol{i}}$, measured about the current wealth:

$$
v_{i}(x)=-u_{i}\left(W_{i}-x\right) .
$$

(4.3) then reads $E\left\{v_{i}\left(\breve{Y}_{i}\right)\right\} \leqslant E\left\{v_{i}\left(Y_{i}\right)\right\}$. Expected disutility for company $i$ (which one wants to keep small!) is denoted by $V_{i}$. We shall assume $v_{i}^{\prime}>0$ and $v_{i}^{\prime \prime}>0$ for all $i$ (risk aversion). As convenient, we shall make transformations of the form $a+b v_{i}(x)$, with $b>0$, which do not affect preference orderings.

Borch (1960a) observed that Pareto-optimality could be obtained for every outcome, and therefore, did not depend upon the distribution of the $X_{i}$. Working with unconstrained REXs (i.e. without $(2.11 \mathrm{~b})$ ), he characterized these solutions as follows:

\section{Theorem 1}

$\operatorname{REX}(X, Y)$ is an unconstrained POREX if and only if there exist positive constants $k_{1}, k_{2}, \ldots, k_{n}$ for which

$$
k_{i} v_{i}^{\prime}\left(Y_{i}\right)=k_{1} v_{1}^{\prime}\left(Y_{1}\right), \quad(i=1,2, \ldots, n) .
$$

A proof is given by DuMouchel (1968). Clearly, there are only $n-1$ effective constants that parametrize the possible POREXs. It also follows that the POREX treaties $Y_{i}=y_{i}\left(X_{1}, X_{1}, \ldots, X_{n}\right)$ are scalar functions of the total losses $X$ only. The values $x$ of $X$ can replace $\omega$ as the "state of the world", and the POREX is hence described by the functions $y_{i}(x),(i=1,2, \ldots, n)$. 
POREXs are pools, in which all losses are first merged, and then divided up. Borch (1968) refers to this result as "non olet" ("l'argent n'a pas d'odeur") with reference to the Vespasian anecdote.

The form of the unconstrained treaties follows easily from (4.5). For example, if the companies all have utility functions that are in one of the following classes (and their positive linear transformations)

$$
u_{i}(x)=\left\{\begin{array} { l } 
{ - e ^ { - a _ { i } x } } \\
{ \pm ( b _ { i } \pm x ) ^ { c } } \\
{ \operatorname { l n } ( x + d _ { i } ) }
\end{array} \left\{\begin{array}{l}
(-,-) \text { for } c>1 \\
(+,+) \text { for } 0<c<1 \\
(-,+) \text { for } c<0
\end{array}\right.\right.
$$

then the POREX sharing rules are linear in the total losses Borch (1968), Wilson (1968)

$$
y_{i}(x)=\beta_{i} x+y_{i}, \Sigma \beta_{i}=1, \Sigma \gamma_{i}=0 .
$$

The quota share fractions $\beta_{i}$ and the side payments $\gamma_{i}$ are determined from the individual utility parameters in (4.6a) as well as the Pareto multipliers from (4.5). Only in the exponential utility case are the quota-sharing fractions independent of the $k_{i}$, and hence the same for all POREXs.

(4.6b) shows a disadvantage of the Borch formulation: because the side payments must sum to zero, there are some companies making payments to others even when all losses are zero. This phenomenon, which one wants to eliminate in certain cases, leads immediately to constrained optimization as considered in the next section.

\subsection{The Constrained Case}

Gerber $(1978)$ was the first to incorporate a non-invasion side constraint and suggested $Y_{i} \leqslant X_{i}+1_{i}$, where the $1_{i}$ are given non-negative constants; this is a special case of our definition of a REX. Gerber also generalized Borch's theorem (4.5) to the constrained case; for our formulation (2.11) it takes the following form:

\section{Theorem 2}

$\operatorname{REX}(X, Y)$ is a constrained POREX if and only if there exist positive constants $k_{1}, k_{2}, \ldots, k_{n}$ and a positive random variable $\Lambda(\omega)$ such that for almost all outcomes $\omega$ and all companies $i=1,2, \ldots, n$

$$
\begin{array}{ll}
k_{i} v_{i}^{\prime}\left(Y_{i}(\omega)\right)=\Lambda(\omega) & \text { if } 0<Y_{i}(\omega)<B_{i}(\omega) \\
k_{i} v_{i}^{\prime}\left(Y_{i}(\omega)\right) \geqslant \Lambda(\omega) & \text { if } Y_{i}(\omega)=0 \\
k_{i} v_{i}^{\prime}\left(Y_{i}(\omega)\right) \leqslant \Lambda(\omega) & \text { if } Y_{i}(\omega)=B_{i}(\omega) .
\end{array}
$$




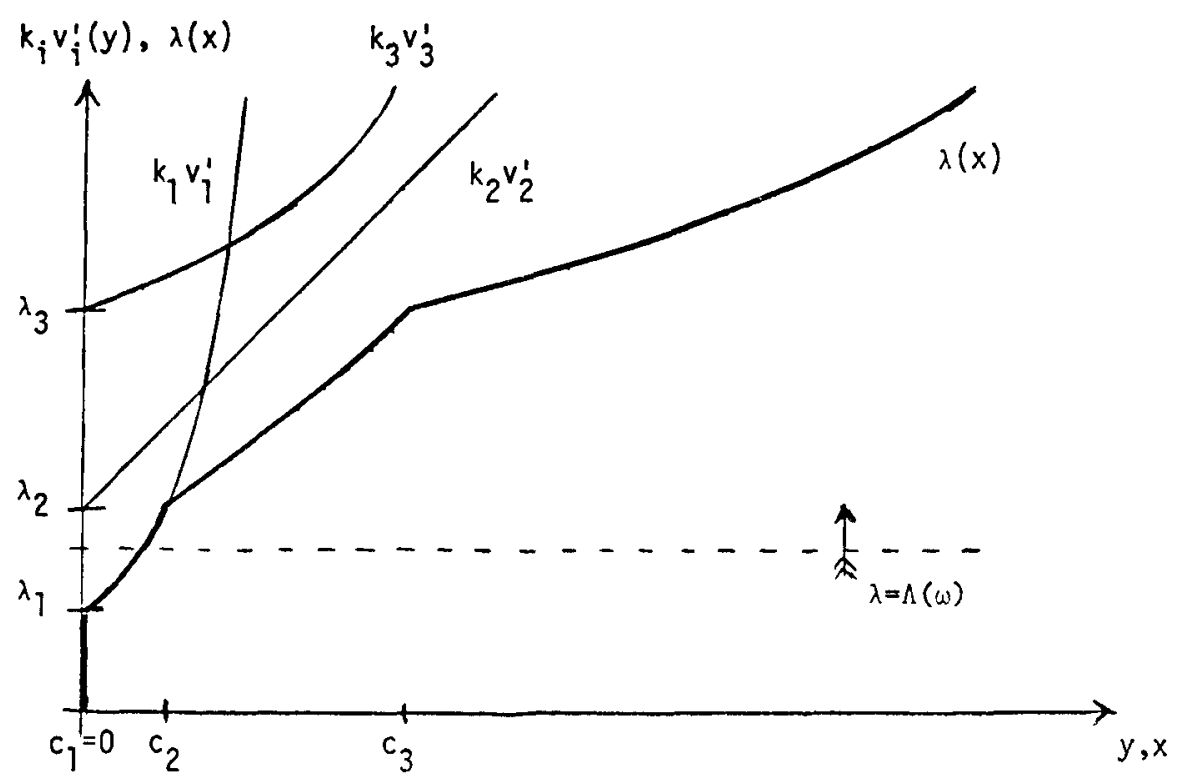

Fig. 1. Vevelopment of a constrained POREX for Given Pareto Multipliers, $h_{i}$.

For an (xplicit proof, the reader is referred to Bühlmann (1978). Observe that for a non-degenerate POREX (i.e. when no company receives a share $Y_{i} \equiv 0$ ), there is exactly one vector $\left(k_{1}, \ldots, k_{n}\right)$ (and its positive multiples) satisfying $(4 \cdot 7)$.

In the following, we shall always assume $B_{i}(\omega) \equiv \infty$ although the case where the upper bound is effective can be treated by similar methods. In particular, Theorem 3 can also be proved for an effective upper bound on the random vector $Y=\left(Y_{1}, Y_{2}, \ldots, Y_{n}\right)$.

\subsection{The Shape of a Constrained POREX}

Condition (4.7) gives us an easy way to visualize and compute the shape of the optimal traties $y_{i}(x)$. The Pareto multipliers $k_{i}$ are fixed arbitrarily, and the curves $k_{i} v_{i}^{\prime}(y)$ are plotted simultaneously for $y>0$ with the level line $\lambda=\Lambda(\omega)$, as shown in Figure 1 for $n=3$. The indices are renumbered to give increasing intercepts $\lambda_{i}=k_{i} v_{i}^{\prime}(0)$.

Now think of $\lambda$ as parametrically increasing from zero. If $\lambda<\lambda_{1}<\lambda_{2}<\lambda_{3}$, it is clear from (4.7) that all $y_{i}$ must equal the lower bound zero, and hence the total losses $x$ are also zero. Increasing $\lambda$ above the first intercept, so that $\lambda_{1}<\lambda<\lambda_{2}<\lambda_{3}$ permits $k_{1} v_{1}^{\prime}\left(y_{1}\right)=\lambda$ and $y_{1}>0$, but still $y_{2}=y_{3}=0$; in other words, the first company takes all the losses $y_{1}=x$. In the next interval, $\lambda_{1}<\lambda_{2}<\lambda<\lambda_{3}, \quad l_{1} v_{1}^{\prime}\left(y_{1}\right)=k_{2} v_{2}^{\prime}\left(y_{2}\right)=\lambda$ and both $y_{1}$ and $y_{2}^{\prime}$ are positive, $y_{1}^{\prime}+y_{2}=x$, but still $y_{3}=0$, and so forth. In other words, by parametrically 
increasing $\lambda$, we pass through different layers of risk-sharing in which first company \# 1, then companies \# 1 and \# 2 , etc., participate.

Furthermore, since at each level $\lambda$ the values of the $y_{i}$ are given directly from the curves, we can compute the total losses $x=\Sigma y_{i}$ by "horizontal addition". The heavy line in Fig. 1 shows the resulting parametric curve of $\lambda$ versus $x$; note the layering constants $c_{1}=0, c_{2}, c_{3}$, at which each new company begins to participate in $x$.

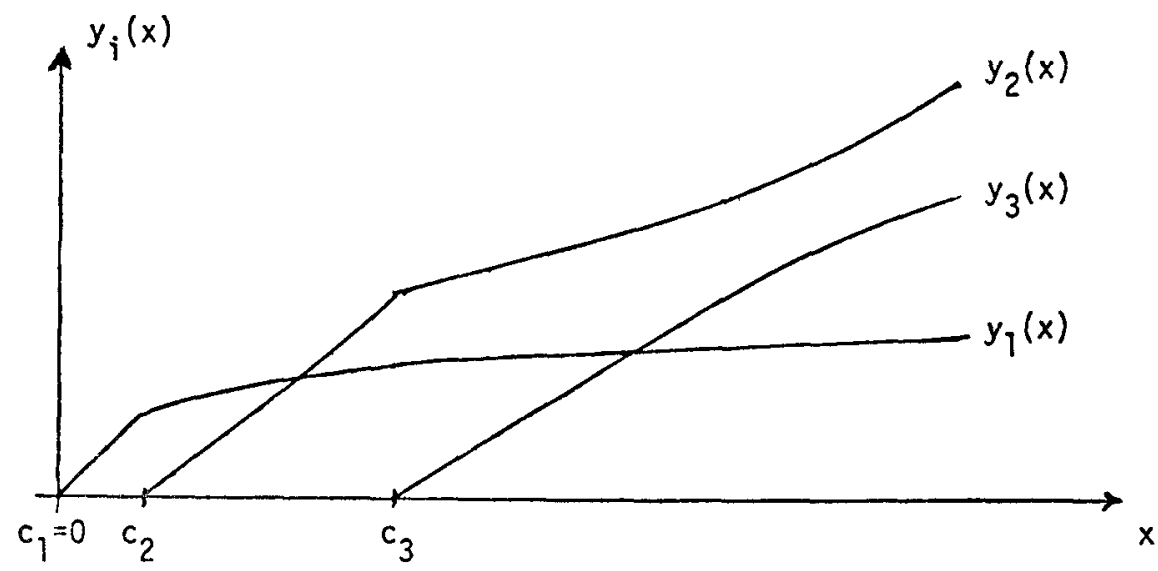

Fig. 2. Layered POREX Treaties corresponding to fig. 1.

By reading fig. 1 sideways, we can easily visualize the fractional participation $y_{i}(x) / x$ at each level $\lambda(x)$; fig. 2 then shows the actual treaties, $y_{i}(x)$ versus $x$, corresponding to fig. 1 .

To summarize, the constrained Pareto-optimal treaties consist of layered, non-linear functions determined parametrically as follows:

1. Given the Pareto multipliers $\left\{k_{i}\right\}$ and the disutility functions $\left\{v_{i}\right\}$, we renumber the companies so that $\lambda_{1} \leqslant \lambda_{2} \leqslant \ldots \leqslant \lambda_{n}$, where $\lambda_{i}=k_{i} v_{i}^{\prime}(0)$. (Take $\lambda_{n+1}=+\infty$ )

2. If $\lambda_{j}<\lambda \leqslant \lambda_{j+1}$, then only companies with indices $i \in J=\{1,2, \ldots, j\}$ participate in the losses, so $y_{i}(\lambda)>o(i \in J)$, and $y_{i}(\lambda) \equiv o(i \notin J),(j=$ $1,2, \ldots, n)$.

3. The participations $y_{i}$ and total losses $x$ in layers $\left(\lambda_{j}, \lambda_{j+1}\right]$ are found from the inverse $w_{i}$ of $v_{i}^{\prime}$ :

$$
y_{i}(\lambda)=w_{i}\left(\frac{\lambda}{k_{i}}\right), \quad(i \in J),
$$

and

$$
x(\lambda)=\sum_{i \in 1} y_{i}(\lambda)
$$

4. Since $v_{i}^{\prime \prime}>0$, this inversion is unique, and the constrained P(OREXs are equal- 
$l_{y}$ characterized by the ordered layering constants $c_{1}=0 \leqslant c_{2} \leqslant c_{3} \leqslant c_{n}$ with

$$
c_{i}=\sum_{j=1}^{i-1} w_{j}\left(\frac{\lambda_{i}}{k_{j}}\right), \quad(i=2, \ldots, n)
$$

Thus, in principle, the participants in a constrained POREX have to determine, through bargaining, the $n-1$ constants which determine the $n$ layers of total pooled loss at which each company will begin to participate; once these values (and their order) are determined, the form of participation is uniquely determined by the individual utility functions. Generally speaking, a first company takes all the loss in the lowest layer, and successive companies start in succeeding layers, with relative participation in higher layers usually (but not necessarily!) diminishing.

\subsection{The Exponential POREX}

Because of simplicity and practical importance, we shall concentrate on exponential (dis)utilities in our later examples, for which all companies have $u_{i}$ proportional to $-e^{-x / \alpha_{i}}$, and, say,

$$
v_{i}(x)=\alpha_{i} e^{+x / \alpha_{i}} ; v_{i}^{\prime}(x)=e^{x i \alpha_{i}}
$$

(This normalization makes $\lambda_{i}=k_{i}$.)

This utility function has the great advantage that the form of the POREX is independent of the initial wealth $W_{i}$ (being absorbed in the $k_{i}$ or the $c_{i}$ );

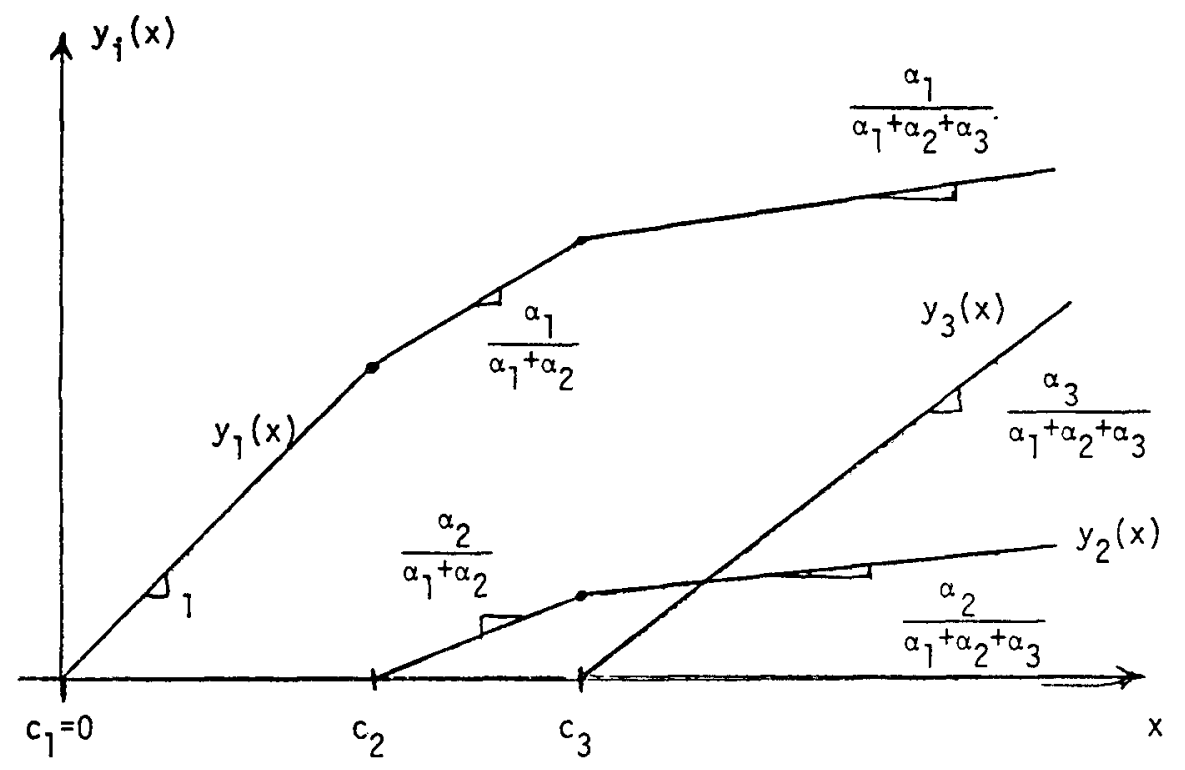

Fig. 3. POREX Treaties with Exponential Utilities. 
this is because the exponential is the only function with constant risk tolerance Wilson (1968) $\alpha_{i}$ is the risk tolerance unit - the larger is $\alpha_{i}$, the more risktolerant is that company.

By simple calculations from the previous subsection (using $\ln v_{i}^{\prime}$ and $\ln \lambda$ ), we find that, given an ordering $(1,2, \ldots, n)$ of the companies and the corresponding layering constants $c_{1}=0 \leqslant c_{2} \leqslant \ldots \leqslant c_{n}$,

$$
y_{i}(x)=\sum_{j=i}^{n} \beta_{i j}\left(x-c_{j}\right)^{+}, \quad(i=1,2, \ldots, n),
$$

with

$$
\beta_{i j}= \begin{cases}0 & (j<i) \\ \alpha_{i} / \sum_{k=1}^{i} \alpha_{k} & (j=i) \\ \alpha_{i}\left[\left(\sum_{k=1}^{j} \alpha_{k}\right)^{-1}-\left(\sum_{k=1}^{j-1} \alpha_{k}\right)^{-1}\right] & (j>i)\end{cases}
$$

independent of the $c_{j}$.

These piecewise-linear treaties are shown in fig. 3 .

Of particular interest are the quota-share fractions ("stock functions" Wilson (1968)), $\beta_{i}(k)$, showing the incremental participation of company $i$ in layer $k$ :

$$
\beta_{i}(k)=\frac{d y_{i}(x)}{d x} \mid x \in\left[c_{k}, c_{k+1}\right)= \begin{cases}0 & (k<i) \\ \alpha_{i} / \sum_{i=1}^{k} \alpha_{j} & (k>i) .\end{cases}
$$

In other words: in the exponential POREX, companies quota-share in layers, with their quota-fraction equal to their unit risk tolerance, divided by unit tolerances of all other companies participating in that layer.

\section{THE FAIR, PARETO-OPTIMAL RISK EXCHANGE (FAIRPOREX)}

We turn now, to the heart of our contribution. The basic difficulty of the POREX models is that they are indeterminate and do not completely describe the "best" treaties. Rather, they prescribe a subset of possible arrangements (which contains any "reasonable" REX), and leave the selection of the layering order and constants as the basic issue in the competitive bargaining. Borch has suggested the use of the Nash equilibrium point Borch (1960a), a market equilibrium mechanism using Arrow Certificates Borch $(1960 \mathrm{~b}, 1962)$ and game theory Borch (1962) to resolve this problem, but none of these is competely satisfactory.

As discussed in Section 3, we believe that mutual exchanges among insurance companies are governed both by a desire to modify short-term risk, and also by an understanding that, over the long run, no single company 
shall profit from another in the sense of modifying the premium of contributed and assumed losses. Therefore, it is of interest to see if the FAIRREX requirement of Section 3 can be superposed on the POREXs of Section 4 to give one or several risk exchanges that are both fair and Pareto-optimal.

First we observe that there are effectively $n-1$ free layering constants $\left\{c_{i}\right\}$ (if we drop the ordering convention), since one must be set to zero.

From the $n$ FAIRREX requirements (3.2) we subtract one because of the already satisfied conservation condition $\Sigma Y_{i}=\Sigma X_{i}$, leaving $n-1$ effective constraints (3.2). Surprisingly, and very satisfyingly, it turns out that this is enough to completely specify a unique FAIRPOREX! Mathematically, this is seen as follows: For any $\operatorname{REX}(X, Y)$ defined in $(2.11)$, let

$$
\Phi(X, Y)=\sum_{i=1}^{n} E\left\{G \cdot \int_{1}^{Y_{i}} \ln v_{i}^{\prime}(y) d y\right\} .
$$

Define a FAIRREX as

where

$$
\begin{gathered}
E\left\{G \cdot y_{i}(X)\right\}=E\left\{G \cdot X_{i}\right\}=q_{i}, \quad(i=1,2, \ldots, n), \\
E\{G X\}=\Sigma q_{i}=q ;
\end{gathered}
$$

and define a FAIRPOREX as a FAIRREX that additionally satisfies (4.7).

\section{Theorem 3}

Suppose $q_{i}>0$ and $v_{i}^{\prime}(0)>0$ for all $i=1,2, \ldots, n$.

Then: For any bounded $X$,

1. Among all FAIRREXs $(X, Y)$ there is a FAIRREX $(X, \widetilde{Y})$ that minimizes $\Phi$;

(5.3) 2. The FAIRREX $(X, \tilde{Y})$ is a FAIRPOREX;

3. If at least one $v_{i}^{\prime \prime}>0$ over its range, then the FAIRPOREX is unique.

The proof is based upon a similar result of Gale (1977) who is concerned with the fair distribution of desirable economic goods. The proof, which relies on the convexity of $\Phi$ on the set of REXs, is rather delicate, and is developed in a separate paper Bühlmann (1978). There one finds also an extension in case $X$ is not bounded.

The practical importance of this result is that a unique POREX can be found which also is fair in the long run, i.e., one can be both a "rational economic man" and a "rational actuary" in setting up a risk exchange.

\section{FAIRPOREX ALGORITHMS}

\subsection{General $n$ Company Algorithm}

In 4.3 we have seen that a POREX can be characterized by its multiplier constants $k=\left(k_{1}, k_{2}, \ldots, k_{n}\right)$ or equivalently by its layering constants 
$c=\left(c_{1}, c_{2}, \ldots, c_{n}\right)$. It is evident that finding a FAIRPOREX is a rootfinding procedure where one tries to find either $k$ or $c$ such that for all $i$

(6.1) $\quad \mu_{i}(c)=\operatorname{Premium}\left[y_{i}(X / c)\right]$ becomes equal to the input premiums $q_{i}=$ Premium $\left[X_{i}\right]$

or, equivalently, such that

(6.2) $\nu_{i}(k)=\operatorname{Premium}\left[y_{i}(X / k)\right]$ becomes equal $q_{i}$.

In the general $n$ company case one works more easily with the multiplier constants. The search is then helped by the following observations:

1. Because of the conservation condition $\Sigma \nu_{i}(k)=\Sigma q_{i}$ for any $k$. So there is no problem of "escaping values" and if one $v_{i}$ is too large at least one other must be too small.

2. If one increases $k_{j}$, then $v_{i}(k)$ is nondecreasing (strictly increasing) for $i \neq j$; is nonincreasing (strictly decreasing) for $i=j$.

The statements in parenthesis hold as soon as $Y_{i}^{(k)}$ is not identically zero.

Observe that these considerations do not depend on any ordering of the constants $k_{j}, j=1,2, \ldots, n$.

\subsection{General Two-Company Algorithm}

The search is particularly simple when $n=2$. Then, for $c_{2}>0, k_{2} \uparrow$ if and only if $c_{2} \uparrow$. If we pick $c^{0}=(0,0)$ initially, then it follows from the above that one $\mu_{i}\left(c^{0}\right)$ is too high, and the other is too low, and that $\mu_{i}$ decreases (increases) if its own (the other) layering constant increases. So the algorithm is simply:

1. Set $c^{0}=(0,0)$ and compute the $\mu_{i}$. Renumber, if necessary, the company with $\mu_{i}>q_{i}$ as company \#2, the other as company \#1.

2. Keeping $c_{1}=0$, increase $c_{2}$ until $\mu_{2}$ decreases to $q_{2}$ (and $\mu_{1}$ increases to $q_{1}$ ).

\subsection{Exponential Utility Algorithm}

The computation of the fair layering constants is greatly simplified in the exponential case because the slope of $y_{i}(x)$ in layer $k, x \in\left[c_{k}, c_{k+1}\right)$, remains constant at $\beta_{i}(k)=\alpha_{i} /\left(\alpha_{1}+\alpha_{2}+\ldots+\alpha_{k}\right)$, and, in the top lay'('r $\left[c_{n}, \infty\right)$, the $n$th company participates only in this layer; thus, $y_{n}(x)=\beta_{n}(n)\left(x-c_{n}\right)^{+}$.

The key idea of the algorithm below is that the company which actually takes on this upper layer will be the one with largest resulting $c_{n}$. Because Premium $\left[(X-c)^{+}\right]$is decreasing in $c$, this company can be found by finding the company $i$ that minimizes $q_{i} / \alpha_{i}$. Once this company is found (and renumbered $\# n)$, the losses handled in the topmost layer are removed from the pool, and the process repeated in the layer $\left[c_{n-1}, c_{n}\right)$, etc. Furthermore, the rankings $q_{i}^{(t)} / \alpha_{i}$, once made, are stable in every iteration $t$, so the ordering of the companies can be fixed once and for all! The algorithm also contains some short-cut stopping rules. 


\section{Exponential Utility Algorithm}

1. Set $t \leftarrow n$, and index the companies so that:

$$
\frac{q_{1}}{\alpha_{1}} \geqslant \frac{q_{2}}{\alpha_{2}} \geqslant \ldots \geqslant \frac{q_{n}}{\alpha_{n}} \text {. }
$$

Define $q_{n}^{(i)}=q_{i}=$ Premium $\left[X_{i}\right]$.

2. At iteration $t, c_{t}$ is the unique solution of

$$
\begin{aligned}
q_{t}^{(t)}=\beta_{t}(t) . & \left\{\text { Premium }\left[\left(X-c_{t}\right)^{+}\right]-\text {Premium }\left[\left(X-c_{t+1}\right)^{+}\right]\right\} \\
& \text {(Note: } \left.c_{n+1}=\infty\right) .
\end{aligned}
$$

3. If $c_{t}=0$ (roundoff ?), STOP! $c_{1}=c_{2}=\ldots=c_{t-1}=0$.

4. Define

$$
\begin{aligned}
q_{i}^{(t-1)}=q_{i}^{(t)}-\beta_{i}(t) \cdot & \left\{\text { Premium }\left[\left(X-c_{t}\right)^{+}\right]-\text {Premium }\left[\left(X-c_{t+1}\right)^{+}\right]\right\}, \\
& (i=1,2, \ldots, t-1) . \\
& \text { (Note: } \left.q_{i}^{(t-1)}=0 \text { for } i \geqslant t\right)
\end{aligned}
$$

5. If $q_{i}^{(t-1)}=0$ (roundoff ?) for all $i<t$, STOP! There is no probability mass below $X=c_{t}$, and $c_{1}=c_{2}=\ldots=c_{t-1}=c_{t}$.

6. Set $t \leftarrow-t-1$. If $t=1$, set $c_{1}=0$ and STOP!

Otherwise, GOTO Step 2.

An equivalent, and somewhat simpler algorithm is as follows:

\section{Alternate Form}

1. Set $t \leftarrow n$, and index the companies so that:

$$
\frac{q_{1}}{\alpha_{1}} \geqslant \frac{q_{2}}{\alpha_{2}} \geqslant \frac{q_{3}}{\alpha_{3}} \geqslant \ldots \geqslant \frac{q_{n}}{\alpha_{n}} .
$$

2. At iteration $t, c_{t}$ is the unique solution of

$$
q_{t}=\beta_{t}(t)\left\{\text { Premium }\left[\left(X-c_{t}\right)^{+}\right]-\sum_{i+1}^{n} q_{i}\right\}
$$

3. If $c_{t}=\mathrm{o}$ (roundoff?), STOP, $c_{1}=c_{2}=\ldots=c_{t-1}=0$.

4. If the term in braces is zero for $c_{t}$, STOP!

There is no probability mass below $X=c_{t}$, and $c_{1}=c_{2}=\ldots=c_{t-1}=c_{t}$.

5. Set $t \leftarrow t-1$. If $t=1$, set $c_{1}=0$ and STOP!

Otherwise, GOTO Step 2.

This algorithm has been implemented in APL for arbitrary $p(x)$ over $X \in[0,1,2, \ldots]$; a copy of the program may be obtained by writing the 
authors. A numeral example, computed according to the first algorithm and using a Pareto distribution is given in Appendix A.

\section{INTERPRETATIONS OF SPECIAL CASES}

With the FAIRPOREX model developed, it is of interest to show how certain practical risk pools arise as special cases. Some of these interpretations have been given previously by Borch (1960a) and Gerber (1978). We shall emphasize the exponential utility case.

The first observation is that with exponential utilities we will have $q_{1} / \alpha_{1}=$ $q_{2} / \alpha_{2}=\ldots=q_{n} / \alpha_{n}$ when the "volume" of each company is the same fraction of its unit risk tolerance. In this case, it is easy to see that $c_{1}=c_{2}=\ldots=c_{n}=0$, and we have a quota claims pool (2.7), with $\theta_{i}=\alpha_{i} /\left(\alpha_{1}+\alpha_{2}+\ldots+\alpha_{n}\right)=$ $q_{i} /\left(q_{1}+q_{2}+\ldots+q_{n}\right)$. All companies participate in all losses, but the most risk-tolerant (highest premium) takes the greatest share; this justifies (2.8). (Of course this result is trivially true if all (general) utility functions are identical and $q_{1}=q_{2}=\ldots=q_{n}$, so $\theta_{i}=n^{-1}$.)

If companies have the same premiums $q_{i}$, but differing $\alpha_{i}$, then they will take higher layers, the larger their risk tolerance is. If both $q_{i}$ and $\alpha_{i}$ vary, then it is the ranking of $q_{i} / \alpha_{i}$ that selects the layering order. It is difficult to give closed formulae for the layers, but one can show that,

$$
\text { Premium }\left[\left(X-c_{t}\right)^{+}\right]-\operatorname{Premium}\left[\left(X-c_{t+1}\right)^{+}\right]=\sum_{i=1}^{i} \alpha_{i}\left[\frac{q_{t}}{\alpha_{t}}-\frac{q_{t+1}}{\alpha_{t+1}}\right]
$$

so the larger $\alpha_{t+1}$, the wider the $t$ th layer, $\left[c_{t}, c_{t+1}\right)$.

As the $\alpha_{n}$ of one company becomes very large compared to the risk tolerance of other companies, it is clear that it will take up the uppermost layer, and take a larger and larger share of it, reaching finally $Y_{n}=1 .\left(X-c_{n}\right)^{+}$, with Premium $\left[\left(X-c_{n}\right)^{+}\right]=q_{n}$. This is, of course, a (full) stop loss cover, with this company acting like a reinsurer. However, we shall see in the next section that such a company might not be interested in participating this pool on fair terms.

The opposite case is a highly risk-adverse company, with $\alpha_{1}$ very small. In the limit $\alpha_{1}=0$ it takes all the losses in $\left[0, c_{1}\right)$, leaving $\left(X-c_{1}\right)^{+}$to the other companies.

If there are many companies participating, then, of course, there are many layers. However, in practice similar companies (with comparable $q_{i} / \alpha_{i}$ ) could probably share the same layers, and lower-level companies could probably be involved in only a few layers (rather than continuing to the end with very small $\beta_{i}(j)$ ), without seriously violating both Pareto-optimality and fairness. This would greatly simplify the administration of the pool. On the other hand our model seems to explain the many practical interlocking hierarchies of pools of different sized carriers. 


\section{THE DESIRABILITY OF FAIRPOREX}

By merging its original loss $X_{i}$ with the pool, company $i$ accepts a new loss $Y_{i}=y_{i}(X)$, where utility is the "best possible" in the Pareto-optimal sense, i.e. for the grand coalition of all companies. Also for all values of $X$, the treaty terms have been adjusted so that the premiums of all companies are unchanged in the long run. But, is it, in the individual sense, always advantageous to join a FAIRPOREX? Unfortunately, the answer is, no.

If we re-examine our development, we see that nowhere was the range of our Pareto-optimal fraction restricted to guarantee that $V_{i}=E\left\{v_{i}\left(Y_{i}\right)\right\} \leqslant$ $V_{i}^{0}=E\left\{v_{i}\left(X_{i}\right)\right\}$ for each participant; in fact, there is no reference to the original marginal distribution (except for the means $q_{i}$ ), and the distribution of the pooled losses $X$ is used only to set the layering constants. The strict requirement of fairness may select a Pareto-optimal solution which has $V_{i}>V_{i}^{\mathbf{0}}$ for some company, who would then prefer to "go it alone". Typically this happens to the larger, risk-tolerant company, who is asked to team up with a smaller, risk-averse company. This forces the larger company to take the tail of the total losses, which may appear worse than not joining the pool.

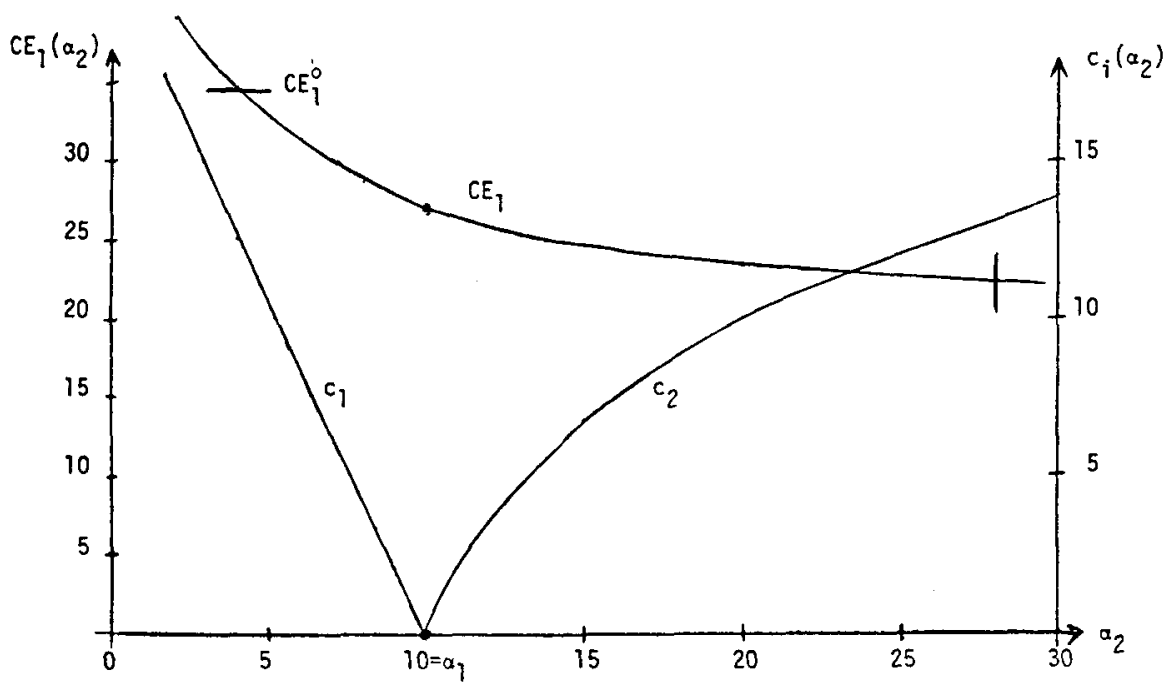

Fig. 4. Numerical Example with $X_{1}, X_{2}$ Geometrically Distributed, $q_{1}=q_{2}=20$, and Exponential Utilities, Showing Layer Values and Certainty Equivalent for Company \#1.

Fig. 4 shows the result of several computer analyses for two companies with exponential disutilities; $\alpha_{1}$ is fixed at 10 units, and $\alpha_{2}$ varies parametrically from 2 to 30 units. The distributions of $X_{1}$ and $X_{2}$ are geometric over the integers, with means $q_{1}=q_{2}=20$ units; $X_{1}$ and $X_{2}$ are assumed independent, so the distribution of $X$ is negative binomial. Thus for $\alpha_{2}<10$, company \#1 
takes the upper layer, with the values of $c_{1}$ shown; for $\alpha_{2}>10$, company \#2 takes the upper layer, with the values of $c_{2}$ shown. ( $c_{i}$ on righthand scale).

Expected disutilities are expressed in terms of certainty equivalents:

$$
v_{i}\left(C E_{i}\right)=E\left\{v_{i}\left(y_{i}(X)\right)\right\} ; v_{i}\left(C E_{i}^{0}\right)=E\left\{v_{i}\left(X_{i}\right)\right\}
$$

or, in the case of exponential disutilities:

$$
C E_{i}=\alpha_{i} \ln E\left\{\exp \left(y_{i}(X) / \alpha_{i}\right)\right\} ; C E_{i}^{0}=\alpha_{i} \ln E\left\{\exp \left(X_{i} / \alpha_{i}\right)\right\}
$$

Without a pool, $C E_{1}^{0}=C E_{2}^{0}=34.14$, i.e. then original random losses are equivalent to a deterministic loss of 34.14 units.

In a pool with $\alpha_{2}=\alpha_{1}=10$, the certainty equivalents drop to $C E_{1}=C E_{2}=$ 24.57 , a large improvement for both companies. Now, let $\alpha_{2}$ decrease, i.e. let company \#2 become more risk-averse. Fig. 4 shows that $C E_{1}$ begins to increase as it is forced to take a larger fraction of a higher layer; finally, at about $\alpha_{2}=4, C E_{1}=C E_{1}^{0}$, and company \# 1 decides the FAIRPOREX is not worth it.

Conversely, if company \# 2 becomes more risk tolerant, then $C E_{1}$ decreases somewhat from 24.57 as $\alpha_{2}$ increases, until finally at about $\alpha_{2}=28$, company \# 2 decides to leave the pool. (Calculations not shown.)

This change in the desirability of a FAIRPOREX can lead to interesting questions of coalition stability with three or more companies. For example, let companies $A, B, C$, each have geometric losses with $q_{A}=q_{B}=q_{C}=10$, and exponential utilities $\alpha_{A}=3, \alpha_{B}=10$, and $\alpha_{C}=20$. Initially, $C E_{A}^{0}=20.53$, $C E_{B}^{0}=13.71$, and $C E_{C}^{0}=11.77$.

Now, through the use of the algorithm, one can show that company $A$ is not acceptable to $B$, and certainly not acceptable to $C$ in a two-party agreement. $B$ and $C$ are compatible, however, and it turns out that: $c_{B}=0, c_{C}=5.19$, $C E_{B}=10.84, C E_{C}=11.48$, when they share the sum of two random geometric losses.

And surprisingly, the three-party exchange is also satisfactory to all parties concerned since: $c_{A}=0, c_{B}=7.07, c_{C}=14.10, C E_{A}=10.38, C E_{B}=11.08$, $C E_{C}=11.72$, when sharing the sum of three random geometric losses.

Note that the "outcast" $A$ gained the most over his initial independent position in the triad, and that $B$ and $C$ were forced to give up some gains from their diad, although they are still better off than going it alone. This suggests that a weak partner should always try to get in at the beginning of the negotiations, and prevent his stronger partners from computing what they can do without him!

We have been unable to form a stable triad from among companies who did not wish to form any diads among themselves. 


\section{THE POSSIBILITY OF SIDE PAYMENTS}

If a FAIRPOREX will not form because of inequities in the sizes of the participants, or because some company feels that it profits insufficiently, a possible generalization of our model is to introduce fixed side payments $\pi_{i}$, such that company $i$ actually pays $\pi_{i}+y_{i}(X)$, with $\Sigma \pi_{i}=0$ and with $y_{i}(X)$ as before. For an exponential utility, this has the quick comparison advantage of increasing the certainty equivalent by $\pi_{i}$. In many cases there is a range of side payments that will bring recalcitrant members back into the exchange, and still leave an improvement in utility for everyone.

For instance, in the example above, $A$ could give 0.24 units to $B$ and 0.24 units to $C$ to induce them to form a triad, and still have a certainty equivalent left of $C E_{A}=10.38+0.48=10.82$, well below being left out at $C E_{A}=20.53$. In fact, he has $20.53-10.82=9.71$ more units of side payment left to "sweeten the pot" if $B$ and $C$ prove stubborn.

Of course, by introducing side payments we abandon the basic idea of fairness. If you want so, this is where the snake bites its own tail. We started by constructing fair risk exchanges and now we modify them to become unfair again!

This just proves that--in our model as in the real world-fair pools will not always form!

\section{APPENDIX A}

\section{Numerical Example (Exponential disutilities)}

1. We demonstrate the calculation of the unconstrained FAIRPOREX (business pool), the constrained FAIRPOREX (claims pool), for a total input variable $X=Z-1$ where $Z$ has density $f_{z}(x)=2 x^{-3},(x \geqslant 1),(Z \sim$ Pareto on $[1, \infty)$ with parameter 3 to guarantee a finite mean $E[Z]=2)$. We also define Premium $[Y]=E[Y]$ for any random variable $Y$.

2. For the unconstrained FAIRPOREX we have [see (4.6)]

$$
y_{i}(x)=\frac{\alpha_{i}}{\sum_{i=1}^{n} \alpha_{j}} x+\gamma_{i}
$$

where $\gamma_{i}$ is computed from the fairness condition

$$
E\left[Y_{i}\right]=q_{i}=>\gamma_{i}=q_{i}-\beta_{i} .
$$

3. For the constrained FAIRPOREX we compute according to the "Exponential Utility Algorithm" (first version) as explained in 6.3. 
THE FAIRPOREX IS COMPUTED For FIVE COMPANIES WITH GIVEN $\alpha_{i}$ AND $q_{i}$ :

\begin{tabular}{|c|c|c|c|c|c|c|c|c|c|c|c|}
\hline$i$ & $\alpha_{i}$ & $q_{i}^{(s)}$ & $\beta_{i}^{(5)}$ & $q_{i}^{(4)}$ & $\beta_{i}^{(4)}$ & $q_{i}^{(3)}$ & $\beta_{i}^{(3)}$ & $q_{i}^{(2)}$ & $\beta_{i}^{(2)}$ & $q_{i}^{(1)}$ & $\beta_{i}^{(1)}$ \\
\hline 5 & 100 & 3 & .585 & 一 & & & & & & & \\
\hline 4 & $5^{\circ}$ & .2 & .292 & .050 & .704 & - & & & & & \\
\hline 3 & 15 & .2 & .088 & .155 & .211 & .140 & .714 & - & & & \\
\hline 2 & 5 & .2 & .029 & .185 & .071 & .180 & .238 & .133 & .833 & - & \\
\hline 1 & 1 & 1 & .006 & .097 & .014 & .096 & .048 & .087 & .167 & .06 & 1 \\
\hline & & \multicolumn{2}{|c|}{$C_{5}=.95$} & \multicolumn{2}{|c|}{$C_{4}=.71$} & \multicolumn{2}{|c|}{$C_{3}=.28$} & \multicolumn{2}{|c|}{$C_{2}=.063$} & \multicolumn{2}{|c|}{$C_{1}=.0007$} \\
\hline
\end{tabular}

Note that in our example

Premium $\left[\left(X-c_{t}\right)^{+}\right]=\frac{1}{c_{t}+1}$

$q_{t}^{(t)}=\beta_{t}(t)\left(\frac{1}{c_{t}+1}-\frac{1}{c_{t+1}+1}\right)=>\frac{1}{c_{t}+1}=\frac{q_{t}^{(t)}}{\beta_{t}^{(t)}}+\frac{1}{c_{t+1}+1}$

and for $i \leqslant t-1$

$q_{i}^{(t-1)}=q_{i}^{(t)}-\beta_{i}(t)\left(\frac{1}{c_{t}+1}-\frac{1}{c_{t+1}+1}\right)$

The computation of $c_{1}$ serves for checking, of course we must have $c_{1}=0$.

4. The results can be rearranged in the following final form:

Business pool

\begin{tabular}{ccc}
\hline \hline company & claims quota & fixed payment (+ pament) \\
(- receipt)
\end{tabular}

Claims pool

\begin{tabular}{crrrcc}
\hline \hline company & layer 5 & layer 4 & layer 3 & layer 2 & layer 1 \\
\hline 5 & $58.8 \%$ & - & - & - & - \\
4 & $29.2 \%$ & $70.4 \%$ & - & - & - \\
3 & $8.8 \%$ & $21.1 \%$ & $71.4 \%$ & - & - \\
2 & $2.9 \%$ & $7.1 \%$ & $23.8 \%$ & $83.3 \%$ & $100 \%$ \\
1 & $0.6 \%$ & $1.4 \%$ & $4.8 \%$ & $16.7 \%$ & \\
\hline layer & & & & & \\
interval & {$[.95, \infty)$} & {$[.71, .95)$} & {$[.28, .71)$} & {$[.063, .28)$} & {$[0, .063)$} \\
\hline
\end{tabular}


REFERENCES

Beard, R. E., Pentikainen, T. and E. Pesonen (1969). Risk Theory, Wiley, New York.

Borch, K. (1960). Reciprocal Reinsurance Treaties, Astin Bulletin, 1, 170-191.

Borch, K. (1960). The Safety Loading of Insurance Premiums, Skandinavisk Aktuarietidskrift, 43, 163-184.

Borch, K. (1962). Equilibrium in a Reinsurance Market, Econometrica, 30, 424-444.

Borch, K. (1968). General Equilibrium in the Economics of Uncertainty, in Risk and Uncertainty, K. Borch and J. Mossin (Eds.), Macmillan, London, 247-264.

Borch, K. (1974). The Mathematical Theory of Insurance, Heath, Lexington.

Bühlmann, H. and W. S. Jewell. Unicity of Fair Pareto-optimal Risk Exchanges, Astin Colloquium 78.

DuMouchel, W. (1968). The Pareto Optimality of an n-Company Reinsurance 'Treaty, Skandinavisk Aktuarietidskrift, 51, 165-170.

GaLe, D. (1977). Fair Division of a Random Harvest, ORC 77-21, Operations Research Center, University of California, Berkeley.

Gerber, H. U. (1978). Pareto-Optimal Risk Exchanges and Related Decision Problems, Astin Bulletin, 10, 25-33.

Lemaire, J. (1977). Echange de Risques Entre Assureurs et Théorie des Jeux, Astin Bulletin, 9, 155-179.

Seat, H. (1969). Stochastic Theory of a Risk Business, Wiley, New York.

Wilson, R. (1968). The Theory of Syndicates, Econometrica, 36, 119-132. 